\title{
New Properties on Degenerate Bell Polynomials
}

\author{
Taekyun Kim $\mathbb{D}^{1},{ }^{1}$ Dae San Kim $\mathbb{D}^{2},{ }^{2}$ Hyunseok Lee $\mathbb{D}^{\mathbb{D}},{ }^{3}$ Seongho Park $\mathbb{D}^{1}$, \\ and Jongkyum Kwon $\mathbb{B D}^{1}$ \\ ${ }^{1}$ Department of Mathematics, Kwangwoon University, Seoul 139-701, Republic of Korea \\ ${ }^{2}$ Department of Mathematics, Sogang University, Seoul 121-742, Republic of Korea \\ ${ }^{3}$ Department of Mathematics Education, Gyeongsang National University, Jinju 52828, Republic of Korea
}

Correspondence should be addressed to Jongkyum Kwon; mathkjk26@gnu.ac.kr

Received 9 September 2021; Revised 9 October 2021; Accepted 15 October 2021; Published 29 October 2021

Academic Editor: Viet-Thanh Pham

Copyright (C) 2021 Taekyun Kim et al. This is an open access article distributed under the Creative Commons Attribution License, which permits unrestricted use, distribution, and reproduction in any medium, provided the original work is properly cited.

The aim of this paper is to study the degenerate Bell numbers and polynomials which are degenerate versions of the Bell numbers and polynomials. We derive some new identities and properties of those numbers and polynomials that are associated with the degenerate Stirling numbers of both kinds.

\section{Introduction}

The Bell number Bel ${ }_{n}$ counts the number of partitions of a set with $n$ elements into disjoint nonempty subsets. The Bell polynomials $\operatorname{Bel}_{n}(x)$, also called Touchard or exponential polynomials, are natural extensions of Bell numbers. The partial and complete Bell polynomials, which are multivariate generalizations of the Bell polynomials, have diverse applications not only in mathematics but also in physics and engineering as well (see [1]).

For instance, the following formula, due to Faà di Bruno formula:

$$
\begin{aligned}
\frac{d^{n}}{\mathrm{~d} t^{n}} f \circ g(\mathrm{t})= & \sum_{k=0}^{n} f^{(k)}(g(t)) B_{n, k} \\
& \left(g^{\prime}(t), g^{\prime \prime}(t), \ldots, g^{(n-k+1)}(t)\right),
\end{aligned}
$$

gives an explicit formula for higher derivatives of composite functions. Here, the partial Bell polynomials $B_{n, k}\left(x_{1}, x_{2}, \ldots\right.$, $\left.x_{n-k+1}\right)$ are defined by

$$
\begin{aligned}
& B_{n, k}\left(x_{1}, x_{2}, \ldots, x_{n-k+1}\right) \\
= & \sum \frac{n !}{\prod_{l=1}^{n-k+1} i_{l} ! \prod_{l=1}^{n-k+1}\left(\frac{x_{l}}{l !}\right)^{i_{l}}, \quad(n \geq k \geq 0),}
\end{aligned}
$$

where the sum runs over all nonnegative integers $i_{1}, i_{2}, \ldots, i_{n-k+1}$, satisfying $i_{1}+i_{2}+\cdots+i_{n-k+1}=k$ and $i_{1}+$ $2 i_{2}+\cdots(n-k+1) i_{n-k+1}=n$ (see [1], p. 133). Then, the complete Bell polynomials are given by $B_{n}\left(x \mid x_{1}, \ldots, x_{n}\right)=$ $\sum_{k=1}^{n} B_{n, k}\left(x_{1}, x_{2}, \ldots, x_{n-k+1}\right) x^{k}, \quad(n \geq 1)$, and $B_{n}(x \mid 1,1$, $\ldots, 1)=\sum_{k=1}^{n} B_{n, k}(1,1, \ldots, 1) x^{k}=\operatorname{Bel}(x), \quad(n \geq 1)$.

As a degenerate version of those Bell polynomials and numbers, the degenerate Bell polynomials $\operatorname{Bel}_{n, \lambda}(x)$ and numbers Bel $_{n, \lambda}$ (see (17)) are introduced and studied under the different names of the partially degenerate Bell polynomials and numbers in [2]. Some interesting identities for them were obtained in connection with Stirling numbers of the first and second kinds [2]. We hope that we will be able to find many interesting applications of these polynomials and numbers in near future. 
In [3], Carlitz initiated the exploration of degenerate Bernoulli and Euler polynomials, which are degenerate versions of the ordinary Bernoulli and Euler polynomials. Along the same line as Carlitz's pioneering work, intensive studies have been done for degenerate versions of quite a few special polynomials and numbers (see [2-10] and the references therein). It is worthwhile to mention that these studies of degenerate versions have been done not only for some special numbers and polynomials but also for transcendental functions like gamma functions (see [8]). The studies have been carried out by various means like combinatorial methods, generating functions, differential equations, umbral calculus techniques, $p$-adic analysis, and probability theory.

The aim of this paper is to further investigate the degenerate Bell polynomials and numbers by means of generating functions. In more detail, we derive several properties and identities of those numbers and polynomials which include recurrence relations for degenerate Bell polynomials (see Theorems 1, 3, 4, and 8), and expressions for them that can be derived from repeated applications of certain operators to the exponential functions (see Theorem 2 , Proposition 1), the derivatives of them (Corollary 1 ), the antiderivatives of them (see Theorem 6), and some identities involving them (see Theorems 5, 9). For the rest of this section, we recall some necessary facts that are needed throughout this paper.

For any $\lambda \in \mathbb{R}$, the degenerate exponential functions are defined by

$$
e_{\lambda}^{x}(t)=\sum_{k=0}^{\infty} \frac{(x)_{k, \lambda}}{k !} t^{k}
$$

(see [9]), where

$$
(x)_{0, \lambda}=1,(x)_{n, \lambda}=x(x-\lambda) \cdots(x-(n-1) \lambda),(n \geq 1) .
$$

When $x=1$, we see use the notation $e_{\lambda}(t)=e_{\lambda}^{1}(t)$.

In [3], Carlitz introduced the degenerate Bernoulli numbers given by

$$
\frac{t}{e_{\lambda}(t)-1}=\sum_{n=0}^{\infty} \beta_{n, \lambda} \frac{t^{n}}{n !}
$$

Note that $\lim _{\lambda \longrightarrow 0} \beta_{n, \lambda}=B_{n}$, where $B_{n}$ are the ordinary Bernoulli numbers given by

$$
\frac{t}{e^{t}-1}=\sum_{n=0}^{\infty} B_{n} \frac{t^{n}}{n !}
$$

(see $[1-14])$.

From (5), we deduce that

$$
\beta_{0, \lambda}=1, \sum_{l=0}^{n-1}\left(\begin{array}{l}
n \\
l
\end{array}\right)(1)_{n-l, \lambda} \beta_{l, \lambda}=0, \quad(n \geq 2),
$$

from which we compute the first few values of $\beta_{n, \lambda}$ as follows:

$$
\begin{aligned}
& \beta_{0, \lambda}=1, \beta_{1, \lambda}=\frac{1}{2} \lambda-\frac{1}{2}, \beta_{2, \lambda}=-\frac{1}{6} \lambda^{2}+\frac{1}{6}, \\
& \beta_{3, \lambda}=\frac{1}{4} \lambda^{3}-\frac{1}{4} \lambda, \beta_{4, \lambda}=-\frac{19}{30} \lambda^{4}+\frac{2}{3} \lambda^{2}-\frac{1}{30}, \\
& \beta_{5, \lambda}=\frac{9}{4} \lambda^{5}-\frac{5}{2} \lambda^{3}+\frac{1}{4} \lambda, \beta_{6, \lambda}=-\frac{863}{84} \lambda^{6}+12 \lambda^{4}-\frac{7}{4} \lambda^{2}+\frac{1}{42}, \\
& \beta_{7, \lambda}=\frac{1375}{24} \lambda^{7}-70 \lambda^{5}+\frac{105}{8} \lambda^{3}-\frac{5}{12} \lambda, \\
& \beta_{8, \lambda}=-\frac{33953}{90} \lambda^{8}+480 \lambda^{6}-\frac{1624}{15} \lambda^{4}+\frac{50}{9} \lambda^{2}-\frac{1}{30} .
\end{aligned}
$$

It is well known that the Stirling numbers of the first kind are defined by

$$
(x)_{n}=\sum_{k=0}^{n} S_{1}(n, k) x^{k}, \frac{1}{k !}(\log (1+t))^{k}=\sum_{n=k}^{\infty} S_{1}(n, k) \frac{t^{n}}{n !},
$$

(see [14]), where $(x)_{0}=1,(x)_{n}=x(x-1) \cdots(x-n+$ $1),(n \geq 1)$.

As the inversion formula of (9), the Stirling numbers of the second kind are given by

$$
x^{n}=\sum_{k=0}^{n} S_{2}(n, k)(x)_{k}, \frac{1}{k !}\left(e^{t}-1\right)^{k}=\sum_{n=k}^{\infty} S_{2}(n, k) \frac{t^{n}}{n !},
$$

(see [14]).

The degenerate Stirling numbers of the first kind are defined by

$$
(x)_{n}=\sum_{k=0}^{n} S_{1, \lambda}(n, k)(x)_{k, \lambda}, \frac{1}{k !}\left(\log _{\lambda}(1+t)\right)^{k}=\sum_{n=k}^{\infty} S_{1, \lambda}(n, k) \frac{t^{n}}{n !},
$$

(see [5]), and the degenerate Stirling numbers of the second kind are given by

$$
(x)_{n, \lambda}=\sum_{k=0}^{n} S_{2, \lambda}(n, k)(x)_{k}, \frac{1}{k !}\left(e_{\lambda}(t)-1\right)^{k}=\sum_{n=k}^{\infty} S_{2, \lambda}(n, k) \frac{t^{n}}{n !},
$$

(see $[5,7])$.

Here, $\log _{\lambda}(1+t)$ is the degenerate logarithm given by

We also recall the degenerate absolute Stirling numbers of the first kind that are defined by

$$
\langle x\rangle_{n}=\sum_{k=0}^{n}\left[\begin{array}{l}
n \\
k
\end{array}\right]_{\lambda}\langle x\rangle_{k, \lambda},
$$

(see [10]), where 


$$
\begin{aligned}
\langle x\rangle_{0} & =1, \quad\langle x\rangle_{n}=x(x+1) \cdots(x+n-1),(n \geq 1), \\
\langle x\rangle_{0, \lambda} & =1, \quad\langle x\rangle_{n, \lambda}=x(x+\lambda)(x+2 \lambda) \cdots(x+(n-1) \lambda),
\end{aligned}
$$

$(n \geq 1)$.

It is well known that the Bell polynomials are defined by

$$
e^{x\left(e^{t}-1\right)}=\sum_{n=0}^{\infty} \operatorname{Bel}_{n}(x) \frac{t^{n}}{n !}
$$

(see $[12,13])$.

When $x=1, \operatorname{Bel}_{n}=\operatorname{Bel}_{n}(1)$ are called the Bell numbers.

From (12), we note that

$$
\operatorname{Bel}_{n}(x)=\sum_{k=0}^{n} S_{2}(n, k) x^{k},
$$

(see $[12,13])$.

In [2], the degenerate Bell polynomials are defined by

$$
e^{x\left(e_{\lambda}(t)-1\right)}=\sum_{n=0}^{\infty} \operatorname{Bel}_{n, \lambda}(x) \frac{t^{n}}{n !} .
$$

Note that $\lim _{\lambda \longrightarrow 0} \operatorname{Bel}_{n, \lambda}(x)=\operatorname{Bel}_{n}(x)$. For $x=1, \operatorname{Bel}_{n, \lambda}=$ $\mathrm{Bel}_{n, \lambda}(1)$ are called the degenerate Bell numbers.
The compositional inverse of $e_{\lambda}(t)$ is given by $\log _{\lambda}(t)$, namely, $e_{\lambda}\left(\log _{\lambda}(t)\right)=t=\log _{\lambda}\left(e_{\lambda}(t)\right)$, where

$$
\log _{\lambda}(1+t)=\frac{1}{\lambda}\left((1+t)^{\lambda}-1\right)=\sum_{n=1}^{\infty} \lambda^{n-1}(1) \frac{1}{n, \frac{t^{n}}{\lambda !}},
$$

(see [5]).

Note that $\lim _{\lambda \longrightarrow 0} \log _{\lambda}(1+t)=\log (1+t)$.

From (17), we note that

$$
\operatorname{Bel}_{n, \lambda}(x)=e^{-x} \sum_{k=0}^{\infty} \frac{(k)_{n, \lambda}}{k !} x^{k}=\sum_{k=0}^{n} S_{2, \lambda}(n, k) x^{k},
$$

(see [2]). by

From (12), we can deduce the recurrence relation given

$$
\begin{array}{r}
S_{2, \lambda}(n+1, k)=S_{2, \lambda}(n, k-1)+(k-n \lambda) S_{2, \lambda}(n, k), \\
(n \geq k \geq 1),
\end{array}
$$

and the values

$$
S_{2, \lambda}(n, 0)=0, \quad(n \geq 1), S_{2, \lambda}(n, n)=1, \quad(n \geq 0),
$$

Now, we compute from (19), (3), and (21) the first few degenerate Bell polynomials as follows:

$$
\begin{aligned}
\operatorname{Bel}_{0, \lambda}(x)= & 1, \operatorname{Bel}_{1, \lambda}(x)=x, \operatorname{Bel}_{2, \lambda}(x)=(-x) \lambda+\left(x^{2}+x\right), \\
\operatorname{Bel}_{3, \lambda}(x)= & (2 x) \lambda^{2}+\left(-3 x^{2}-3 x\right) \lambda+\left(x^{3}+3 x^{2}+x\right), \\
\operatorname{Bel}_{4, \lambda}(x)= & (-6 x) \lambda^{3}+\left(11 x^{2}+12 x\right) \lambda^{2}+\left(-6 x^{3}-18 x^{2}-7 x\right) \lambda+\left(x^{4}+6 x^{3}+7 x^{2}+x\right), \\
\operatorname{Bel}_{5, \lambda}(x)= & (24 x) \lambda^{4}+\left(-50 x^{2}-60 x\right) \lambda^{3}+\left(35 x^{3}+110 x^{2}+50 x\right) \lambda^{2}+\left(-10 x^{4}-60 x^{3}-75 x^{2}-15 x\right) \lambda \\
& +\left(x^{5}+10 x^{4}+25 x^{3}+15 x^{2}+x\right), \\
\operatorname{Bel}_{6, \lambda}(x)= & (-120 x) \lambda^{5}+\left(274 x^{2}+274 x\right) \lambda^{4}+\left(-225 x^{3}-675 x^{2}-225 x\right) \lambda^{3} \\
& +\left(85 x^{4}+510 x^{3}+595 x^{2}+85 x\right) \lambda^{2}+\left(-15 x^{5}-150 x^{4}-375 x^{3}-225 x^{2}-15 x\right) \lambda \\
& +\left(x^{6}+15 x^{5}+65 x^{4}+90 x^{3}+31 x^{2}+x\right), \\
\operatorname{Bel}_{7, \lambda}(x)= & (720 x) \lambda^{6}+\left(-1764 x^{2}-1764 x\right) \lambda^{5}+\left(1624 x^{3}+4872 x^{2}+1624 x\right) \lambda^{4} \\
& +\left(-735 x^{4}-4410 x^{3}-5145 x^{2}-735 x\right) \lambda^{3}+\left(175 x^{5}+1750 x^{4}+4375 x^{3}+2625 x^{2}+175 x\right) \lambda^{2} \\
& +\left(-21 x^{6}-315 x^{5}-1365 x^{4}-1890 x^{3}-651 x^{2}-21 x\right) \lambda+\left(x^{7}+21 x^{6}+140 x^{5}+350 x^{4}+301 x^{3}+63 x^{2}+x\right), \\
\operatorname{Bel}_{8, \lambda}(x)= & (-5040 x) \lambda^{7}+\left(13068 x^{2}+13068 x\right) \lambda^{6}+\left(-13132 x^{3}-39396 x^{2}-13132 x\right) \lambda^{5} \\
& +\left(6769 x^{4}+40614 x^{3}+47383 x^{2}+6769 x\right) \lambda^{4}+\left(-1960 x^{5}-19600 x^{4}-49000 x^{3}-29400 x^{2}-1960 x\right) \lambda^{3} \\
& +\left(332 x^{6}+4830 x^{5}+20930 x^{4}+28980 x^{3}+9982 x^{2}+322 x\right) \lambda^{2} \\
& +\left(-28 x^{7}-588 x^{6}-3920 x^{5}-9800 x^{4}-8428 x^{3}-1764 x^{2}-28 x\right) \lambda \\
& +\left(x^{8}+28 x^{7}+266 x^{6}+1050 x^{5}+1701 x^{4}+966 x^{3}+127 x^{2}+x\right) .
\end{aligned}
$$



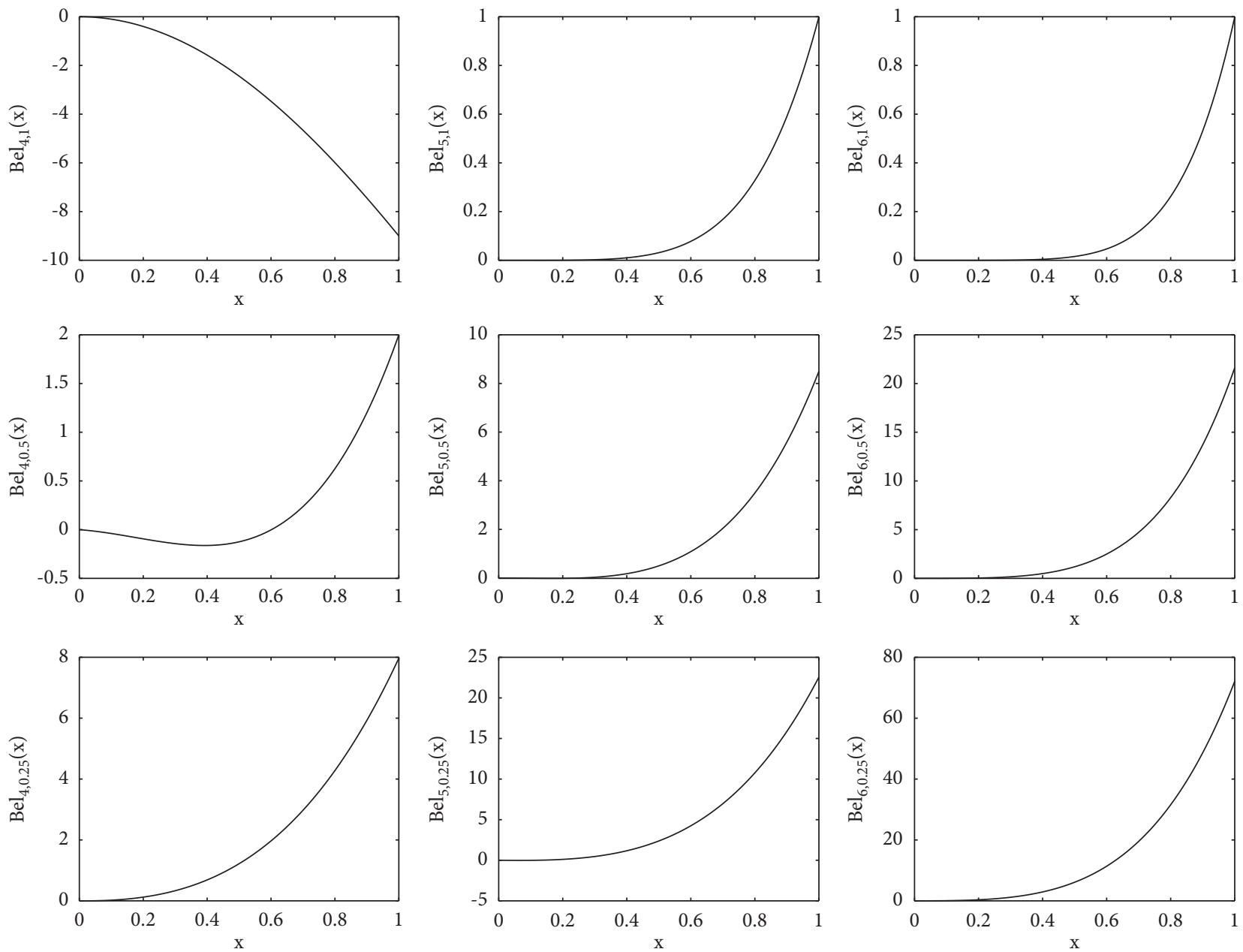

Figure 1: The shapes of Bell polynomials $\operatorname{Bel}_{k, \lambda}(x)$.

In Figure 1, we plot the shapes of Bell polynomial Bel $k, \lambda(x)$. The upper-left graph looks different from the others. However, of course they all go to infinity as $x$ tends to infinity.

\section{Some New Properties on Degenerate \\ Bell Polynomials}

Let $a$ be a nonzero constant. First, we observe that

$$
\begin{aligned}
\frac{d^{n}}{\mathrm{~d} t^{n}} e^{a\left(e_{\lambda}(t)\right)} & =\frac{d^{n}}{d t^{n}} \sum_{k=0}^{\infty} \frac{a^{k}}{k !} e_{\lambda}^{k}(t)=\sum_{k=0}^{\infty} \frac{a^{k}}{k !}(k)_{n, \lambda} e_{\lambda}^{k-n \lambda}(t) \\
& =\sum_{k=0}^{\infty} \frac{(k)_{n, \lambda}}{k !} a^{k} e_{\lambda}^{k}(t) \frac{1}{(1+\lambda t)^{n}} \\
& =\left(\sum_{k=0}^{\infty} \frac{(k)_{n, \lambda}}{k !}\left(a e_{\lambda}(t)\right)^{k} e^{-a e_{\lambda}(t)}\right) e^{a e_{\lambda}(t)} \frac{1}{(1+\lambda t)^{n}} \\
& =\frac{1}{(1+\lambda t)^{n}} \operatorname{Bel}_{n, \lambda}\left(a e_{\lambda}(t)\right) e^{a e_{\lambda}(t)} .
\end{aligned}
$$

Therefore, by (23), we obtain the following lemma.
Lemma 1. For $n \geq 0$, the $n$th derivative of $e^{a\left(e_{\lambda}(t)\right)}$ is given by

$$
\frac{d^{n}}{\mathrm{~d} t^{n}} e^{a\left(e_{\lambda}(t)\right)}=\frac{1}{(1+\lambda t)^{n}} \operatorname{Bel}_{n, \lambda}\left(a e_{\lambda}(t)\right) e^{a e_{\lambda}(t)} .
$$

Let $x=e_{\lambda}(t)$ in (23). Then, we have

$$
\frac{d}{\mathrm{~d} t}=\frac{\mathrm{d} x}{\mathrm{~d} t} \frac{d}{\mathrm{~d} x}=\frac{1}{1+\lambda t} e_{\lambda}(t) \frac{d}{\mathrm{~d} x}=x^{1-\lambda} \frac{d}{\mathrm{~d} x} .
$$

By Lemma 1 and (25), we get

$$
\left(x^{1-\lambda} \frac{d}{\mathrm{~d} x}\right)^{n} e^{a x}=x^{-n \lambda} \operatorname{Bel}_{n, \lambda}(a x) e^{a x}, \quad(n \geq 0) .
$$

Let

$$
S_{n, \lambda}=\sum_{k=0}^{\infty} \frac{(k)_{n, \lambda}}{k !}, \quad n=0,1,2, \ldots
$$

Then, we note from (19) that we have

$$
e \operatorname{Bel}_{n, \lambda}=S_{n, \lambda} .
$$

The generating function of $S_{n, \lambda}$ is given by 


$$
e^{e_{\lambda}(t)}=\sum_{n=0}^{\infty} S_{n, \lambda} \frac{t^{n}}{n !}
$$

Indeed, this can be seen from the following:

$$
\sum_{n=0}^{\infty} S_{n, \lambda} \frac{t^{n}}{n !}=e \sum_{n=0}^{\infty} \operatorname{Bel}_{n, \lambda}(1) \frac{t^{n}}{n !}=e e^{e_{\lambda}(t)-1}=e^{e_{\lambda}(t)} .
$$

Taking the derivative with respect to $t$ on both sides of (30), we have

$$
\begin{aligned}
\sum_{n=0}^{\infty} S_{n+1, \lambda} \frac{t^{n}}{n !} & =\frac{d}{\mathrm{~d} t} e^{e_{\lambda}(t)}=e_{\lambda}^{1-\lambda}(t) e^{e_{\lambda}(t)} \\
& =\sum_{l=0}^{\infty}(1-\lambda)_{l, \lambda} \frac{t^{l}}{l !} \sum_{m=0}^{\infty} S_{m, \lambda} \frac{t^{m}}{m !} \\
& =\sum_{n=0}^{\infty}\left(\sum_{m=0}^{n}\left(\begin{array}{c}
n \\
m
\end{array}\right) S_{m, \lambda}(1-\lambda)_{n-m, \lambda}\right) \frac{t^{n}}{n !} \\
& =\sum_{n=0}^{\infty}\left(\sum_{m=0}^{n}\left(\begin{array}{c}
n \\
m
\end{array}\right) S_{m, \lambda}(1)_{n-m+1, \lambda}\right) \frac{t^{n}}{n !} .
\end{aligned}
$$

Thus, by comparing the coefficients on both sides of (31) and from (28), we obtain the following theorem.

Theorem 1. For $n \geq 0$, the following recurrence relation holds:

$$
\operatorname{Bel}_{n+1, \lambda}=\sum_{m=0}^{n}\left(\begin{array}{c}
n \\
m
\end{array}\right) \operatorname{Bel}_{m, \lambda}(1)_{n-m+1, \lambda} .
$$

Assume that the following identity holds:

$$
\left(x^{1-\lambda} \frac{d}{\mathrm{~d} x}\right)^{n} e^{x}=\sum_{k=0}^{\infty} \frac{(k)_{n, \lambda}}{k !} x^{k-n \lambda}
$$

Then, we have

$$
\begin{aligned}
\left(x^{1-\lambda} \frac{d}{\mathrm{~d} x}\right)^{n+1} e^{x} & =x^{1-\lambda} \frac{d}{\mathrm{~d} x} \sum_{k=0}^{\infty} \frac{(k)_{n, \lambda}}{k !} x^{k-n \lambda} \\
& =x^{1-\lambda} \sum_{k=0}^{\infty} \frac{(k)_{n, \lambda}}{k !}(k-n \lambda) x^{k-n \lambda-1} \\
& =\sum_{k=0}^{\infty} \frac{(k)_{n+1, \lambda}}{k !} x^{k-(n+1) \lambda} .
\end{aligned}
$$

This together with (26) gives the next result.

Theorem 2. For $n \geq 0$, the following relations hold true:

$$
\left(x^{1-\lambda} \frac{d}{\mathrm{~d} x}\right)^{n} e^{x}=\sum_{k=0}^{\infty} \frac{(k)_{n, \lambda}}{k !} x^{k-n \lambda}=x^{-n \lambda} \operatorname{Bel}_{n, \lambda}(x) e^{x} .
$$

From the first equality in (35) and (27), we see that we have

$$
S_{n, \lambda}=\left.\left(x^{1-\lambda} \frac{d}{\mathrm{~d} x}\right)^{n} e^{x}\right|_{x=1} .
$$

Clearly, $S_{0, \lambda}=S_{1, \lambda}=e$. We can check that

$$
\begin{aligned}
\left(x^{1-\lambda} \frac{d}{\mathrm{~d} x}\right)^{2} e^{x} & =(1-\lambda) x^{1-2 \lambda} e^{x}+x^{2-2 \lambda} e^{x} \\
\left(x^{1-\lambda} \frac{d}{\mathrm{~d} x}\right)^{3} e^{x} & =x^{1-3 \lambda} e^{x}\left((1-\lambda)_{2, \lambda}+(1-\lambda) x\right) \\
& +x^{2-3 \lambda} e^{x}(2-2 \lambda+x) .
\end{aligned}
$$

From (36) and (37), we have $S_{2, \lambda}=(2-\lambda) e$, $S_{3, \lambda}=\left(2 \lambda^{2}-6 \lambda+5\right) e$.

By taking $x(d / \mathrm{d} x)$ in the second equality of (35), on the one hand, we have

$$
x \frac{d}{\mathrm{~d} x}\left(x^{-n \lambda} \operatorname{Bel}_{n, \lambda}(x) e^{x}\right)=\sum_{k=0}^{\infty} \frac{(k)_{n+1, \lambda}}{k !} x^{k-n \lambda} .
$$

On the other hand, we also have

$$
\begin{aligned}
x \frac{d}{\mathrm{~d} x}\left(x^{-n \lambda} \operatorname{Bel}_{n, \lambda}(x) e^{x}\right)= & x x^{-n \lambda}\left(\operatorname{Bel}_{n, \lambda}^{\prime}(x)+\operatorname{Bel}_{n, \lambda}(x)\right) e^{x} \\
& -n \lambda x^{-n \lambda} \operatorname{Bel}_{n, \lambda}(x) e^{x},
\end{aligned}
$$

where $\operatorname{Bel}_{n, \lambda}^{\prime}(x)=(d / \mathrm{d} x) \operatorname{Bel}_{n, \lambda}(x)$.

From (38) and (39) and Theorem 2, we note that

$$
\begin{aligned}
\sum_{k=0}^{\infty} \frac{(k)_{n+1, \lambda}}{k !} x^{k} & =\left(\sum_{k=0}^{\infty} \frac{(k)_{n+1, \lambda}}{k !} x^{k} e^{-x}\right) e^{x}=\operatorname{Bel}_{n+1, \lambda}(x) e^{x} \\
& =x\left(\operatorname{Bel}_{n, \lambda}{ }^{\prime}(x)+\operatorname{Bel}_{n, \lambda}(x)\right) e^{x}-n \lambda \operatorname{Bel}_{n, \lambda}(x) e^{x} .
\end{aligned}
$$

Therefore, by (40) and Theorem 2, we obtain the following theorem.

Theorem 3. For $n \geq 0$, the following identity holds:

$$
\operatorname{Bel}_{n+1, \lambda}(x)=x\left(\operatorname{Bel}_{n, \lambda}^{\prime}(x)+\operatorname{Bel}_{n, \lambda}(x)\right)-n \lambda \operatorname{Bel}_{n, \lambda}(x),
$$

where $B e l_{n, \lambda}^{\prime}(x)=(d / d x) B e l_{n, \lambda}(x)$.

From (17), we note that 


$$
\begin{aligned}
\sum_{n=0}^{\infty} \frac{d}{\mathrm{~d} x} \operatorname{Bel}_{n, \lambda}(x) \frac{t^{n}}{n !} & =\frac{\partial}{\partial x} e^{x\left(e_{\lambda}(t)-1\right)}=\left(e_{\lambda}(t)-1\right) e^{x\left(e_{\lambda}(t)-1\right)} \\
& =\left(\sum_{l=0}^{\infty}(1)_{l, \lambda} \frac{t^{l}}{l !}-1\right) \sum_{m=0}^{\infty} \operatorname{Bel}_{m, \lambda}(x) \frac{t^{m}}{m !} \\
& =\sum_{n=0}^{\infty}\left(\sum_{m=0}^{n}\left(\begin{array}{c}
n \\
m
\end{array}\right) \operatorname{Bel}_{m, \lambda}(x)(1)_{n-m, \lambda}-\operatorname{Bel}_{n, \lambda}(x)\right) \frac{t^{n}}{n !} \\
& =\sum_{n=0}^{\infty}\left(\sum_{m=0}^{n-1}\left(\begin{array}{c}
n \\
m
\end{array}\right) \operatorname{Bel}_{m, \lambda}(x)(1)_{n-m, \lambda}\right) \frac{t^{n}}{n !} .
\end{aligned}
$$

Thus, by comparing the coefficients on both sides of (42), we get

$$
\frac{d}{\mathrm{~d} x} \operatorname{Bel}_{n, \lambda}(x)=\operatorname{Bel}_{n, \lambda}^{\prime}(x)=\sum_{m=0}^{n-1}\left(\begin{array}{c}
n \\
m
\end{array}\right) \operatorname{Bel}_{m, \lambda}(x)(1)_{n-m, \lambda},
$$$$
(n \geq 1) \text {. }
$$

Taking the derivative with respect to $t$ on both sides of (17), we have

$$
\frac{d}{\mathrm{~d} t} e^{x\left(e_{\lambda}(t)-1\right)}=\sum_{n=0}^{\infty} \operatorname{Bel}_{n+1, \lambda}(x) \frac{t^{n}}{n !}
$$

On the other hand,

$$
\begin{aligned}
\frac{d}{\mathrm{~d} t} e^{x\left(e_{\lambda}(t)-1\right)} & =x e_{\lambda}^{1-\lambda}(t) e^{x\left(e_{\lambda}(t)-1\right)} \\
& =x \sum_{l=0}^{\infty}(1-\lambda)_{l, \lambda} \frac{t^{l}}{l !} \sum_{m=0}^{\infty} \operatorname{Bel}_{m, \lambda}(x) \frac{t^{m}}{m !} \\
& =x \sum_{n=0}^{\infty}\left(\sum_{m=0}^{n}\left(\begin{array}{c}
n \\
m
\end{array}\right) \operatorname{Bel}_{n, \lambda}(x)(1-\lambda)_{n-m, \lambda}\right) \frac{t^{n}}{n !} \\
& =\sum_{n=0}^{\infty}\left(x \sum_{m=0}^{n}\left(\begin{array}{c}
n \\
m
\end{array}\right) \operatorname{Bel}_{m, \lambda}(x)(1)_{n-m+1, \lambda}\right) \frac{t^{n}}{n !} .
\end{aligned}
$$

Therefore, by (44) and (45), we obtain the following theorem.

Theorem 4. . For $n \geq 0$, the following recurrence relation is valid:

$$
\operatorname{Bel}_{n+1, \lambda}(x)=x \sum_{m=0}^{n}\left(\begin{array}{l}
n \\
m
\end{array}\right) \operatorname{Bel}_{m, \lambda}(x)(1)_{n-m+1, \lambda} .
$$

Remark 1 . Theorems 3 and 4 and (43) give us the following:

$$
\begin{aligned}
\operatorname{Bel}_{n+1, \lambda}(x) & =x \sum_{m=0}^{n}\left(\begin{array}{c}
n \\
m
\end{array}\right) \operatorname{Bel}_{m, \lambda}(x)(1)_{n-m, \lambda}-n \lambda \operatorname{Bel}_{n, \lambda}(x) \\
& =x \sum_{m=0}^{n}\left(\begin{array}{l}
n \\
m
\end{array}\right) \operatorname{Bel}_{m, \lambda}(x)(1)_{n-m, \lambda}(1-(n-m) \lambda) .
\end{aligned}
$$

This implies that the following identity must hold true:

$$
n \operatorname{Bel}_{n, \lambda}(x)=x \sum_{m=0}^{n}\left(\begin{array}{l}
n \\
m
\end{array}\right)(n-m) \operatorname{Bel}_{m, \lambda}(x)(1)_{n-m, \lambda},
$$

the validity of which follows from Theorem 4 .

From Theorem 3, we note that

$$
\begin{aligned}
x \operatorname{Bel}_{n, \lambda}^{\prime}(x)= & x \frac{d}{\mathrm{~d} x} \operatorname{Bel}_{n, \lambda}(x)=\operatorname{Bel}_{n+1, \lambda}(x)-x \operatorname{Bel}_{n, \lambda}(x) \\
& +n \lambda \operatorname{Bel}_{n, \lambda}(x) \\
= & \operatorname{Bel}_{n+1, \lambda}(x)-(x-n \lambda) \operatorname{Bel}_{n, \lambda}(x) \\
= & x \sum_{m=0}^{n-1}\left(\begin{array}{c}
n \\
m
\end{array}\right) \operatorname{Bel}_{m, \lambda}(x)(1)_{n+1-m, \lambda}+n \lambda \operatorname{Bel}_{n, \lambda}(x) .
\end{aligned}
$$

Therefore, by (49), we obtain the following corollary.

Corollary 1. For $n \geq 1$, we have the following identity:

$$
x \frac{d}{\mathrm{~d} x} \operatorname{Bel}_{n, \lambda}(x)=x \sum_{m=0}^{n-1}\left(\begin{array}{c}
n \\
m
\end{array}\right) \operatorname{Bel}_{m, \lambda}(x)(1)_{n+1-m, \lambda}+n \lambda \operatorname{Bel}_{n, \lambda}(x) .
$$

We observe that 


$$
\begin{aligned}
x^{1-\lambda} \frac{d}{\mathrm{~d} x}\left(x^{-n \lambda} \operatorname{Bel}_{n, \lambda}(x) e^{x}\right) & =x^{1-\lambda} \frac{d}{\mathrm{~d} x}\left(x^{-n \lambda} \sum_{k=0}^{\infty} \frac{(k)_{n, \lambda}}{k !} x^{k}\right) \\
& =\sum_{k=0}^{\infty} \frac{(k)_{n+1, \lambda}}{k !} x^{k-(n+1) \lambda} \\
& =x^{-(n+1) \lambda}\left(\sum_{k=0}^{\infty} \frac{(k)_{n+1, \lambda}}{k !} x^{k} e^{-x}\right) e^{x} \\
& =x^{-(n+1) \lambda} \operatorname{Bel}_{n+1, \lambda}(x) e^{x}, \quad(n \geq 0) .
\end{aligned}
$$

Thus, by (51), we get

$x^{1-\lambda} \frac{d}{\mathrm{~d} x}\left(x^{-n \lambda} \operatorname{Bel}_{n, \lambda}(x) e^{x}\right)=x^{-(n+1) \lambda} \operatorname{Bel}_{n+1, \lambda}(x) e^{x}, \quad(n \geq 0)$.

From (17), we have

$$
\begin{aligned}
\sum_{n=0}^{\infty} \operatorname{Bel}_{n, \lambda}(x+y) \frac{t^{n}}{n !} & =e^{(x+y)\left(e_{\lambda}(t)-1\right)}=e^{x\left(e_{\lambda}(t)-1\right)} \cdot e^{y\left(e_{\lambda}(t)-1\right)} \\
& =\sum_{l=0}^{\infty} \operatorname{Bel}_{l, \lambda}(x) \frac{t^{l}}{l !} \sum_{m=0}^{\infty} \operatorname{Bel}_{m, \lambda}(x) \frac{t^{m}}{m !} \\
& =\sum_{n=0}^{\infty}\left(\sum_{l=0}^{n}\left(\begin{array}{l}
n \\
l
\end{array}\right) \operatorname{Bel}_{l, \lambda}(x) \operatorname{Bel}_{n-l, \lambda}(y)\right) \frac{t^{n}}{n !}
\end{aligned}
$$

Therefore, by comparing the coefficients on both sides of (53), we obtain the following theorem.

Theorem 5. For $n \geq 0$, the following binomial identity holds:

$$
\operatorname{Bel}_{n, \lambda}(x+y)=\sum_{l=0}^{n}\left(\begin{array}{l}
n \\
l
\end{array}\right) \operatorname{Bel}_{l, \lambda}(x) \operatorname{Bel}_{n-l, \lambda}(y) .
$$

From (17), we note that

$$
\sum_{n=0}^{\infty} \int_{0}^{x} \operatorname{Bel}_{n, \lambda}(x) \mathrm{d} x \frac{t^{n}}{n !}=\int_{0}^{x} e^{x\left(e_{\lambda}(t)-1\right)} \mathrm{d} x .
$$

On the other hand, we also have

$$
\begin{aligned}
\int_{0}^{x} e^{x\left(e_{\lambda}(t)-1\right)} \mathrm{d} x & =\frac{1}{e_{\lambda}(t)-1}\left[e^{x\left(e_{\lambda}(t)-1\right)}\right]_{0}^{x} \\
& =\frac{1}{e_{\lambda}(t)-1}\left(e^{x\left(e_{\lambda}(t)-1\right)}-1\right)=\frac{1}{e_{\lambda}(t)-1} \sum_{k=1}^{\infty} \operatorname{Bel}_{k, \lambda}(x) \frac{t^{k}}{k !} \\
& =\frac{t}{e_{\lambda}(t)-1} \sum_{k=0}^{\infty} \frac{\operatorname{Bel}_{k+1, \lambda}(x)}{k+1} \frac{t^{k}}{k !}=\sum_{l=0}^{\infty} \beta_{l, \lambda} \frac{t^{l}}{l !} \sum_{k=0}^{\infty} \frac{\operatorname{Bel}_{k+1, \lambda}(x) t^{k}}{k+1} \frac{t^{k}}{k !} \\
& =\sum_{n=0}^{\infty}\left(\sum_{k=0}^{n}\left(\begin{array}{c}
n \\
k
\end{array}\right) \frac{\operatorname{Bel}_{k+1, \lambda}(x)}{k+1} \beta_{n-k, \lambda}\right) \frac{t^{n}}{n !} \\
& =\sum_{n=0}^{\infty}\left(\frac{1}{n+1} \sum_{k=0}^{n}\left(\begin{array}{c}
n+1 \\
k+1
\end{array}\right) \operatorname{Bel}_{k+1, \lambda}(x) \beta_{n-k, \lambda}\right) \frac{t^{n}}{n !} \\
& =\sum_{n=0}^{\infty}\left(\frac{1}{n+1} \sum_{k=1}^{n+1}\left(\begin{array}{c}
n+1 \\
k
\end{array}\right) \operatorname{Bel}_{k, \lambda}(x) \beta_{n+1-k, \lambda}\right) \frac{t^{n}}{n !}
\end{aligned}
$$


Therefore, by (55) and (56), we obtain the following theorem.

Theorem 6. For $n \geq 0$, the antiderivative of $B e l_{n, \lambda}(x)$ is given by

$$
\int_{0}^{x} \operatorname{Bel}_{n, \lambda}(x) \mathrm{d} x=\frac{1}{n+1} \sum_{k=1}^{n+1}\left(\begin{array}{c}
n+1 \\
k
\end{array}\right) \beta_{n+1-k, \lambda} \operatorname{Bel}_{k, \lambda}(x),
$$

By comparing the coefficients on both sides of (58), we have

$\frac{1}{k !} \sum_{j=0}^{k}\left(\begin{array}{l}k \\ j\end{array}\right)(-1)^{k-j}(j)_{n, \lambda}= \begin{cases}S_{2, \lambda}(n, k), & \text { if } n \geq k, \\ 0, & \text { if } \$ 0 \leq n \leq k-1 \$ .\end{cases}$

Let $D=(d / \mathrm{d} x)$, and let $y=x^{p}$. As $x^{1-\lambda} D=$ $p y^{1-(\lambda / p)}(d / \mathrm{d} y)$, we have

where $\beta_{n, \lambda}$ are Carlitz's degenerate Bernoulli numbers given by $\left(t /\left(e_{\lambda}(t)-1\right)\right)=\sum_{n=0}^{\infty} \beta_{n, \lambda}\left(t^{n} / n !\right)$.

For $k \geq 0$, by (12), we get

$$
\begin{aligned}
\sum_{n=k}^{\infty} S_{2, \lambda}(n, k) \frac{t^{n}}{n !} & =\frac{1}{k !}\left(e_{\lambda}(t)-1\right)^{k}=\frac{1}{k !} \sum_{j=0}^{k}(-1)^{k-j} e_{\lambda}^{j}(t)\left(\begin{array}{l}
k \\
j
\end{array}\right) \\
& =\sum_{n=0}^{\infty}\left(\frac{1}{k !} \sum_{j=0}^{k}\left(\begin{array}{l}
k \\
j
\end{array}\right)(-1)^{k-j}(j)_{n, \lambda}\right) \frac{t^{n}}{n !} .
\end{aligned}
$$

$$
\begin{aligned}
\left(x^{1-\lambda} D\right)^{n} e^{a x^{p}} & =\left(p y^{1-(\lambda / p)} \frac{d}{\mathrm{~d} y}\right)^{n} e^{a y}=p^{n}\left(y^{1-(\lambda / p)} \frac{d}{\mathrm{~d} y}\right)^{n} e^{a y} \\
& =p^{n} y^{-(n \lambda / p)} \operatorname{Bel}_{n,(\lambda / p)}(a y) e^{a y}=p^{n} x^{-n \lambda} \operatorname{Bel}_{n,(\lambda / p)}\left(a x^{p}\right) e^{a x^{p}}
\end{aligned}
$$

Thus, we have

$$
\left(x^{1-\lambda} D\right)^{n} e^{a x^{p}}=p^{n} x^{-n \lambda} \operatorname{Bel}_{n,(\lambda / p)}\left(a x^{p}\right) e^{a x^{p}}, \quad(n \geq 0) .
$$

Proposition 1. For $n \geq 0$, we have the following operational formula:

$$
x^{n \lambda}\left(x^{1-\lambda} D\right)^{n} e^{a x^{p}}=p^{n} \operatorname{Bel}_{n,(\lambda / p)}\left(a x^{p}\right) e^{a x^{p}},
$$

Therefore, by (61), we obtain the following proposition. where $D=(d / d x)$.

From (12), we note that

$$
\begin{aligned}
\sum_{k=0}^{n+1} S_{2, \lambda}(n+1, k)(x)_{k} & =(x)_{n+1, \lambda}=(x-n \lambda)(x)_{n, \lambda} \\
& =(x-n \lambda) \sum_{k=0}^{n} S_{2, \lambda}(n, k)(x)_{k}=\sum_{k=0}^{n} S_{2, \lambda}(n, k)(x-k+k-n \lambda)(x)_{k} \\
& =\sum_{k=0}^{n} S_{2, \lambda}(n, k)(x)_{k+1}+\sum_{k=0}^{n} S_{2, \lambda}(n, k)(k-n \lambda)(x)_{k} \\
& =\sum_{k=1}^{n+1} S_{2, \lambda}(n, k-1)(x)_{k}+\sum_{k=0}^{n} S_{2, \lambda}(n, k)(k-n \lambda)(x)_{k} \\
& =\sum_{k=0}^{n+1}\left(S_{2, \lambda}(n, k-1)+S_{2, \lambda}(n, k)(k-n \lambda)\right)(x)_{k} .
\end{aligned}
$$


By (63), we get

$S_{2, \lambda}(n+1, k)=S_{2, \lambda}(n, k-1)+(k-n \lambda) S_{2, \lambda}(n, k)$,

where $0 \leq k \leq n+1$.

We prove the next theorem by induction on $n$.

Theorem 7. Assume that $f$ is an infinitely differentiable function. Then, for $n \geq 0$, the following operational formula holds:

$$
\left(x^{1-\lambda} D\right)^{n} f=\sum_{k=0}^{n} S_{2, \lambda}(n, k) x^{k-n \lambda} D^{k} f
$$

where $D=(d / d x)$.

Proof. The statement is obviously true for $n=0$. Assume that it is true for $n,(n \geq 0)$.

$$
\begin{aligned}
\left(x^{1-\lambda} D\right)^{n+1} f(x) & =\left(x^{1-\lambda} D\right) \sum_{k=0}^{n} S_{2, \lambda}(n, k) x^{k-n \lambda} D^{k} f(x) \\
& =x^{1-\lambda} \sum_{k=0}^{n} S_{2, \lambda}(n, k)\left\{(k-n \lambda) x^{k-1-n \lambda} D^{k} f(x)+x^{k-n \lambda} D^{k+1} f(x)\right\} \\
& =\sum_{k=0}^{n} S_{2, \lambda}(n, k)\left\{(k-n \lambda) x^{k-(n+1) \lambda} D^{k} f(x)+x^{k+1-(n+1) \lambda} D^{k+1} f(x)\right\} \\
& =\sum_{k=0}^{n+1} S_{2, \lambda}(n, k)(k-n \lambda) x^{k-(n+1) \lambda} D^{k} f(x)+\sum_{k=0}^{n+1} S_{2, \lambda}(n, k-1) x^{k-(n+1) \lambda} D^{k} f(x) \\
& =\sum_{k=0}^{n+1}\left\{S_{2, \lambda}(n, k)(k-n \lambda)+S_{2, \lambda}(n, k-1)\right\} x^{k-(n+1) \lambda} D^{k} f(x) \\
& =\sum_{k=0}^{n+1} S_{2, \lambda}(n+1, k) x^{k-(n+1) \lambda} D^{k} f(x) .
\end{aligned}
$$

Let $f(x)=e^{x}$. Then, we have

$$
\begin{aligned}
x^{n \lambda}\left(x^{1-\lambda} D\right)^{n} e^{x} & =\left(\sum_{k=0}^{n} S_{2, \lambda}(n, k) x^{k}\right) e^{x} \\
& =\operatorname{Bel}_{n, \lambda}(x) e^{x} .
\end{aligned}
$$

Observe that, for any $\alpha$, we have

$$
\left(x^{1-\lambda} D\right)^{n} x^{\alpha}=(\alpha)_{n, \lambda} x^{\alpha-n \lambda}
$$

By the Leibniz rule, we get

$$
\left(x^{1-\lambda} D\right)^{n}(f g)=\sum_{l=0}^{n}\left(\begin{array}{l}
n \\
l
\end{array}\right)\left[\left(x^{1-\lambda} D\right)^{n-l} f\right]\left[\left(x^{1-\lambda} D\right)^{l} g\right] \text {. }
$$

$$
\begin{aligned}
x^{-(n+m) \lambda} e^{x} \operatorname{Bel}_{n+m, \lambda}(x) & =\left(x^{1-\lambda} D\right)^{n+m} e^{x}=\left(x^{1-\lambda} D\right)^{n}\left(x^{1-\lambda} D\right)^{m} e^{x} \\
& =\left(x^{1-\lambda} D\right)^{n}\left(x^{-m \lambda} \operatorname{Bel}_{m, \lambda}(x) e^{x}\right) .
\end{aligned}
$$

By (69) and (70), we get

$$
\begin{aligned}
& x^{-(n+m) \lambda} e^{x} \operatorname{Bel}_{n+m, \lambda}(x)=\left(x^{1-\lambda} D\right)^{n}\left(x^{-m \lambda} \operatorname{Bel}_{m, \lambda} e^{x}\right) \\
& =\sum_{k=0}^{n}\left(\begin{array}{l}
n \\
k
\end{array}\right)\left[\left(x^{1-\lambda} D\right)^{n-k}\left(x^{-m \lambda} \operatorname{Bel}_{m, \lambda}(x)\right)\right]\left[\left(x^{1-\lambda} D\right)^{k} e^{x}\right] \\
& =\sum_{k=0}^{n}\left(\begin{array}{l}
n \\
k
\end{array}\right) x^{-k \lambda} \operatorname{Bel}_{k, \lambda}(x) e^{x}\left[\left(x^{1-\lambda} D\right)^{n-k}\left(x^{-m \lambda} \operatorname{Bel}_{m, \lambda}(x)\right)\right] .
\end{aligned}
$$

(69) On the other hand,

From Theorem 2, we note that

$$
\begin{aligned}
\left(x^{1-\lambda} D\right)^{n-k}\left(x^{-m \lambda} \operatorname{Bel}_{m, \lambda}(x)\right) & =\sum_{j=0}^{m} S_{2, \lambda}(m, j)\left[\left(x^{1-\lambda} D\right)^{n-k} x^{j-m \lambda}\right] \\
& =\sum_{j=0}^{m} S_{2, \lambda}(m, j)(j-m \lambda)_{n-k, \lambda} x^{j-m \lambda-(n-k) \lambda}=\sum_{j=0}^{m} S_{2, \lambda}(m, j)(j-m \lambda)_{n-k, \lambda} x^{j-(m+n) \lambda+k \lambda} \\
& =\sum_{j=0}^{m} S_{2, \lambda}(m, j) \frac{(j)_{m+n-k, \lambda}}{(j)_{m, \lambda}} x^{j-(m+n) \lambda+k \lambda}
\end{aligned}
$$


By (71) and (72), we get

$$
\begin{aligned}
& x^{-(n+m) \lambda} e^{x} \operatorname{Bel}_{n+m, \lambda}(x) \\
& =\sum_{k=0}^{n}\left(\begin{array}{l}
n \\
k
\end{array}\right) x^{-k \lambda} \operatorname{Bel}_{k, \lambda}(x) e^{x} \sum_{j=0}^{m} S_{2, \lambda}(m, j) \frac{(j)_{m+n-k, \lambda}}{(j)_{m, \lambda}} x^{j-(m+n) \lambda+k \lambda} \\
& =x^{-(m+n) \lambda} e^{x} \sum_{k=0}^{n} \sum_{j=0}^{m}\left(\begin{array}{l}
n \\
k
\end{array}\right) S_{2, \lambda}(m, j) \operatorname{Bel}_{k, \lambda}(x) \frac{(j)_{m+n-k, \lambda}}{(j)_{m, \lambda}} x^{j}
\end{aligned}
$$

Therefore, by comparing the coefficients on both sides of (73), we obtain the following theorem.

Theorem 8. For $m, n \geq 0$, we have the following expression:

$$
\operatorname{Bel}_{n+m, \lambda}(x)=\sum_{k=0}^{n} \sum_{j=0}^{m}\left(\begin{array}{l}
n \\
k
\end{array}\right) S_{2, \lambda}(m, j) \operatorname{Bel}_{k, \lambda}(x) \frac{(j)_{m+n-k, \lambda}}{(j)_{m, \lambda}} x^{j} .
$$

Taking $x=1$ in (74), we have

$$
\operatorname{Bel}_{n+m, \lambda}=\sum_{k=0}^{n} \sum_{j=0}^{m}\left(\begin{array}{l}
n \\
k
\end{array}\right) S_{2, j}(m, j) \operatorname{Bel}_{k, \lambda} \frac{(j)_{n+m-k, \lambda}}{(j)_{m, \lambda}} .
$$

From (19) and (68), we note that

$$
\begin{aligned}
\left(x^{1-\lambda} D\right)^{n} \operatorname{Bel}_{m, \lambda}(x) & =\left(x^{1-\lambda} D\right)^{n} \sum_{k=0}^{m} S_{2, \lambda}(m, k) x^{k} \\
& =\sum_{k=0}^{m} S_{2, \lambda}(m, k)(k)_{n, \lambda} x^{k-n \lambda} .
\end{aligned}
$$
we get

On the other hand, by Leibniz rule (69) and Theorem 2,

$$
\begin{aligned}
\left(x^{1-\lambda} D\right)^{n} \operatorname{Bel}_{m, \lambda}(x) & =\left(x^{1-\lambda} D\right)^{n}\left[\left(e^{-x} x^{m \lambda}\right)\left(\operatorname{Bel}_{m, \lambda}(x) e^{x} x^{-m \lambda}\right)\right] \\
& =\sum_{k=0}^{n}\left(\begin{array}{l}
n \\
k
\end{array}\right)\left[\left(x^{1-\lambda} D\right)^{n-k}\left(x^{m \lambda} e^{-x}\right)\right]\left[\left(x^{1-\lambda} D\right)^{k}\left(\operatorname{Bel}_{m, \lambda}(x) e^{x} x^{-m \lambda}\right)\right] \\
& =\sum_{k=0}^{n}\left(\begin{array}{l}
n \\
k
\end{array}\right)\left[\left(x^{1-\lambda} D\right)^{n-k}\left(x^{m \lambda} e^{-x}\right)\right]\left[\left(x^{1-\lambda} D\right)^{m+k} e^{x}\right] \\
& =\sum_{k=0}^{n}\left(\begin{array}{l}
n \\
k
\end{array}\right)\left[\left(x^{1-\lambda} D\right)^{n-k}\left(x^{m \lambda} e^{-x}\right)\right] x^{-(m+k) \lambda} \operatorname{Bel}_{m+k, \lambda}(x) e^{x} .
\end{aligned}
$$

By (68) and (69) and Theorem 2, we easily get

$$
\begin{aligned}
\left(x^{1-\lambda} D\right)^{n-k}\left(x^{m \lambda} e^{-x}\right) & =\sum_{j=0}^{n-k}\left(\begin{array}{c}
n-k \\
j
\end{array}\right)\left[\left(x^{1-\lambda} D\right)^{j} x^{m \lambda}\right]\left[\left(x^{1-\lambda} D\right)^{n-k-j} e^{-x}\right] \\
& =\sum_{j=0}^{n-k}\left(\begin{array}{c}
n-k \\
j
\end{array}\right)(m \lambda)_{j, \lambda} x^{m \lambda-j \lambda} e^{-x} \operatorname{Bel}_{n+k-j, \lambda}(-x) x^{-(n-k-j) \lambda} \\
& =\sum_{j=0}^{n-k}\left(\begin{array}{c}
n-k \\
j
\end{array}\right)(m \lambda)_{j, \lambda} x^{m \lambda-n \lambda+k \lambda} \operatorname{Bel}_{n-k-j, \lambda}(-x) e^{-x} .
\end{aligned}
$$


From (77) and (78), we have

$$
\begin{aligned}
& \left(x^{1-\lambda} D\right)^{n} \operatorname{Bel}_{m, \lambda}(x) \\
& =\sum_{k=0}^{n}\left(\begin{array}{l}
n \\
k
\end{array}\right) x^{-m \lambda-k \lambda} \operatorname{Bel}_{m+k, \lambda}(x) e^{x} \sum_{j=0}^{n-k}\left(\begin{array}{c}
n-k \\
j
\end{array}\right)(m \lambda)_{j, \lambda} x^{m \lambda-n \lambda+k \lambda} \operatorname{Bel}_{n-k-j, \lambda}(-x) e^{-x} \\
& =\sum_{k=0}^{n} \sum_{j=0}^{n-k}\left(\begin{array}{l}
n \\
k
\end{array}\right)\left(\begin{array}{c}
n-k \\
j
\end{array}\right) \operatorname{Bel}_{m+k, \lambda}(x) \operatorname{Bel}_{n-k-j, \lambda}(-x)(m \lambda)_{j, \lambda} x^{-n \lambda} .
\end{aligned}
$$

Therefore, by (76) and (79), we obtain the following theorem.

Theorem 9. For $m, n \geq 0$, the following identity holds true.

$$
\begin{aligned}
& \sum_{k=0}^{m} S_{2, \lambda}(m, k)(k)_{n, \lambda} x^{k} \\
& =\sum_{k=0}^{n} \sum_{j=0}^{n-k}\left(\begin{array}{l}
n \\
k
\end{array}\right)\left(\begin{array}{c}
n-k \\
j
\end{array}\right) \operatorname{Bel}_{m+k, \lambda}(x) \operatorname{Bel}_{n-k-j, \lambda}(-x)(m \lambda)_{j, \lambda} .
\end{aligned}
$$

By (11) and (13), we easily get

$$
(-1)^{n-k} S_{1, \lambda}(n, k)=\left[\begin{array}{l}
n \\
k
\end{array}\right]_{\lambda}, \quad(0 \leq k \leq n) .
$$

Indeed,

$$
\begin{aligned}
\sum_{n=0}^{\infty}\langle x\rangle_{n} \frac{t^{n}}{n !} & =\left(\frac{1}{1-t}\right)^{x}=e_{\lambda}^{-x}\left(\log _{\lambda}(1-t)\right) \\
& =\sum_{k=0}^{\infty}(-x)_{k, \lambda} \frac{1}{k !}\left(\log _{\lambda}(1-t)\right)^{k} \\
& =\sum_{k=0}^{\infty}(-1)^{k}\langle x\rangle_{k, \lambda} \sum_{n=k}^{\infty} S_{1, \lambda}(n, k) \frac{(-t)^{n}}{n !} \\
& =\sum_{n=0}^{\infty}\left(\sum_{k=0}^{n}(-1)^{n-k} S_{1, \lambda}(n, k)\langle x\rangle_{k, \lambda}\right) \frac{t^{n}}{n !}
\end{aligned}
$$

Therefore, by (82), we get

$$
\langle x\rangle_{n}=\sum_{k=0}^{n}(-1)^{n-k} S_{1, \lambda}(n, k)\langle x\rangle_{k, \lambda} .
$$

Replacing $t$ by $\log _{\lambda}(1+t)$ in (17), we get

$$
\begin{aligned}
e^{x t} & =\sum_{k=0}^{\infty} \operatorname{Bel}_{k, \lambda}(x) \frac{1}{k !}\left(\log _{\lambda}(1+t)\right)^{k} \\
& =\sum_{k=0}^{\infty} \operatorname{Bel}_{k, \lambda}(x) \sum_{n=k}^{\infty} S_{1, \lambda}(n, k) \frac{t^{n}}{n !} \\
& =\sum_{n=0}^{\infty}\left(\sum_{k=0}^{n} \operatorname{Bel}_{k, \lambda}(x) S_{1, \lambda}(n, k)\right) \frac{t^{n}}{n !} .
\end{aligned}
$$

Thus, from (81) and (84), we get

$$
x^{n}=\sum_{k=0}^{n} \operatorname{Bel}_{k, \lambda}(x)(-1)^{n-k}\left[\begin{array}{l}
n \\
k
\end{array}\right]_{\lambda} .
$$

From (13), we note that

$$
\begin{aligned}
\sum_{k=0}^{n+1}\left[\begin{array}{c}
n+1 \\
k
\end{array}\right]_{\lambda}\langle x\rangle_{k, \lambda} & =\langle x\rangle_{n+1}=(x+n)\langle x\rangle_{n} \\
& =(x+n) \sum_{k=0}^{n}\left[\begin{array}{l}
n \\
k
\end{array}\right]_{\lambda}\langle x\rangle_{k, \lambda} \\
& =\sum_{k=0}^{n}\left[\begin{array}{l}
n \\
k
\end{array}\right]_{\lambda}(x+k \lambda+n-k \lambda)\langle x\rangle_{k, \lambda} \\
& =\sum_{k=0}^{n}\left[\begin{array}{l}
n \\
k
\end{array}\right]_{\lambda}\langle x\rangle_{k+1, \lambda}+\sum_{k=0}^{n}(n-k \lambda)\left[\begin{array}{l}
n \\
k
\end{array}\right]_{\lambda}\langle x\rangle_{k, \lambda} \\
& =\sum_{k=0}^{n+1}\left[\begin{array}{c}
n \\
k-1
\end{array}\right]_{\lambda}\langle x\rangle_{k, \lambda}+\sum_{k=0}^{n+1}(n-k \lambda)\left[\begin{array}{l}
n \\
k
\end{array}\right]_{\lambda}\langle x\rangle_{k, \lambda} \\
& =\sum_{k=0}^{n+1}\left(\left[\begin{array}{c}
n \\
k-1
\end{array}\right]_{\lambda}+(n-k \lambda)\left[\begin{array}{l}
n \\
k
\end{array}\right]_{\lambda}\right)_{\langle x\rangle_{k, \lambda} .}
\end{aligned}
$$

Thus, by comparing the coefficients on both sides of (86), we get

$$
\left[\begin{array}{c}
n+1 \\
k
\end{array}\right]_{\lambda}=\left[\begin{array}{c}
n \\
k-1
\end{array}\right]_{\lambda}+(n-k \lambda)\left[\begin{array}{l}
n \\
k
\end{array}\right]_{\lambda}, \quad(0 \leq k \leq n+1) .
$$

\section{Conclusion}

Here, we studied by means of generating functions the degenerate Bell polynomials which are degenerate versions of the Bell polynomials. In more detail, we derived recurrence relations for degenerate Bell polynomials (see Theorems $1,3,4$, and 8), and expressions for them that can be derived from repeated applications of certain operators to the exponential functions (see Theorem 2 and Proposition 1 ), the derivatives of them (Corollary 1 ), the antiderivatives of them (see Theorem 6), and some identities involving them (see Theorems 5 and 9).

As one of our future projects, we would like to continue to study degenerate versions of certain special polynomials and numbers and their applications to physics, science, and 
engineering as well as to mathematics. An earlier version of this paper has been presented as preprint in [15].

\section{Data Availability}

No data were used to support this study.

\section{Disclosure}

An earlier version of the paper has been presented as preprint in the following link: https://arxiv.org/abs/2108.06260.

\section{Conflicts of Interest}

The authors declare that they have no conflicts of interest.

\section{Acknowledgments}

This work was supported by the National Research Foundation of Korea (NRF) grant funded by the Korea government (No. 2020R1F1A1A01071564).

\section{References}

[1] L. Comtet, Advanced Combinatorics: The Art of Finite and Infinite Expansions, Springer, Dordrecht, Netherlands, 1974.

[2] T. Kim, D. S. Kim, and D. V. Dolgy, "On partially degenerate Bell numbers and polynomials," Proceedings of the Jangjeon Mathematical Society, vol. 20, no. 3, pp. 337-345, 2017.

[3] L. Carlitz, "Degenerate stirling, Bernoulli and eulerian numbers," Utilitas Mathematica, vol. 15, pp. 51-88, 1979.

[4] M. Acikgoz and U. Duran, "Unified degenerate central Bell polynomials," Journal of Mathematical Analysis, vol. 11, no. 2, pp. 18-33, 2020.

[5] D. S. Kim and T. Kim, "A note on a new type of degenerate Bernoulli numbers," Russian Journal of Mathematical Physics, vol. 27, no. 2, pp. 227-235, 2020.

[6] H. K. Kim, "Fully degenerate Bell polynomials associated with degenerate Poisson random variables," Open Mathematics, vol. 19, no. 1, pp. 284-296, 2021.

[7] T. Kim, "A note on degenerate stirling polynomials of the second kind," Proceedings of the Jangjeon Mathematical Society, vol. 20, no. 3, pp. 319-331, 2017.

[8] T. Kim and D. S. Kim, "Note on the degenerate gamma function," Russian Journal of Mathematical Physics, vol. 27, no. 3, pp. 352-358, 2020.

[9] T. Kim and D. S. Kim, "Degenerate zero-truncated Poisson random variables," Russian Journal of Mathematical Physics, vol. 28 , no. 1 , pp. 66-72, 2021.

[10] T. Kim, D. S. Kim, H. Lee, and J.-W. Park, "A note on degenerate $r$-stirling numbers," Journal of Inequalities and Applications, vol. 225, p. 12, 2020.

[11] S. Araci, M. Acikgoz, and E. Sen, "Some new formulae for Genocchi numbers and polynomials involving Bernoulli and Euler polynomials," International Journal of Mathematics and Mathematical Sciences, vol. 2014, Article ID 760613, 7 pages, 2014.

[12] E. T. Bell, "Exponential numbers," The American Mathematical Monthly, vol. 41, no. 7, pp. 411-419, 1934.

[13] K. N. Boyadzhiev, "Exponential polynomials, stirling numbers, and evaluation of some gamma integrals," Abstract and Applied Analysis, vol. 2009, Article ID 168672, 18 pages, 2009.
[14] S. Roman, "The umbral calculus," Pure and Applied Mathematics, Academic Press, Inc. [Harcourt Brace Jovanovich, Publishers], New York, NY, USA, 1984.

[15] T. Kim, D. S. Kim, H. Lee, and S. Park, "Some new properties on degenerate bell polynomials," Preprint. 